\title{
Generalized Invexity of Higher Order and Its Applications in Variational Problems
}

\author{
Promila Kumar' ${ }^{1}$, Jyoti ${ }^{2}$ \\ ${ }^{1}$ Gargi College, University of Delhi, New Delhi, India \\ ${ }^{2}$ Department of Mathematics, University of Delhi, Delhi, India \\ Email: kumar.promila@gmail.com, deepshahj_p@yahoo.co.in
}

Received 18 July 2015; accepted 22 August 2015; published 25 August 2015

Copyright (C) 2015 by authors and Scientific Research Publishing Inc.

This work is licensed under the Creative Commons Attribution International License (CC BY).

http://creativecommons.org/licenses/by/4.0/

(c) (i) Open Access

\begin{abstract}
In the present paper the definition of invexity for continuous functions is extended to invexity of order $\boldsymbol{m}$ which is further generalized to $\rho$-pseudoinvexity type I of order $\boldsymbol{m}, \rho$-pseudoinvexity type II of order $m$, as well as $\rho$-quasi invexity type I of order $m$ and $\rho$-quasiinvexity type II of order $m$. The central objective of the paper is to study variational problem where the functionals involved satisfy the above stated generalized $\rho$-invexity assumptions of order $m$. Wolfe type and Mond Weir type of duals are formulated. Sufficient optimality conditions and duality results are proved. It is demonstrated with the help of an example that the class of $\rho$-invex functionals of order $m$ is more general than the class of $\rho$-invex functionals. Further, it may be noted that the results presented in this paper are more powerful than the existing results as this new class of functions defined here satisfies $\boldsymbol{m}^{\text {th }}$ derivative test.
\end{abstract}

\section{Keywords}

Optimality, Variational Problem, Higher Order $\rho$-Invexity, Duality

\section{Introduction}

Calculus of variations is a powerful technique to find solutions of various problems appearing in dynamics of rigid bodies, optimization of orbits, theory of vibrations and many other fields. The subjects whose importance is fast growing in science and engineering primarily concern with finding optimal value of a definite integral involving certain functions subject to fixed boundary value conditions. In [1] Courant and Hilbert quoted an earlier work of Friedrichs [2] and gave a dual relationship for a simple type of unconstrained variational problem. Subsequently, Hanson [3] pointed out that some of the duality results of mathematical programming have analogues in variational calculus. Exploring this relationship between mathematical programming and the classical 
calculus of variation, Mond and Hanson [4] formulated a constrained variational problem as a mathematical programming problem and proved optimality and duality results under the assumption of convexity. To weaken this notion of convexity, Hanson [5] defined Invex (invariably convex) functions.

Invexity plays a vital role in many aspects of mathematical programming and hence in calculus of variation. Invexity was extended to variational problems by Mond, Chandra and Husain [6]. To relax invexity assumption imposed on the functions involved, various generalized notions have been proposed. One of the useful generalizations was $\rho$-invexity given by Bhatia and Kumar [7]. Another generalization of invexity namely B-vexity for variational problems was also given by Bhatia and Kumar [8]. Concept of higher order derivative for variational problems was studied by Husain and Jabeen [9] while generalized type-1 invexity and duality were explored by Kim and Kim [10]. Bhatia and Sahay [11] introduced higher order strong invexity for multiobjective optimization problem for static case. In this paper we extend the notion of $\rho$-invexity for continuous functions to higher order $\rho$-invexity and utilize this to establish sufficient optimality conditions for variational problem. Wolfe and Mond-Weir type duals are also formulated and various duality theorems are proved under the assumption of generalized $\rho$-invexity of higher order. The present concepts of invexity in the continuous case lead to four types of generalizations of invexity whereas normally only two types of generalizations are presented in literature. The significance of this new notion of invexity allows us to relax the notion of invexity associated with optimality and duality results for the variational problem.

\section{Preliminaries and Definitions}

Let $R^{n}$ denote n-dimensional Euclidean space and $I=[a, b]$ be a real interval. Let $f: I \times R^{n} \times R^{n} \rightarrow R$ and $g: I \times R^{n} \times R^{n} \rightarrow R^{p}$ be functions with continuous derivative up to and including second order with respect to each of their arguments. Let $X$ be the space of continuously differentiable state functions, $x: I \rightarrow R^{n}$ with its derivative $\dot{x}$ such that $x(a)=\alpha, x(b)=\beta$ and it is equipped with the norm $\|x\|=\|x\|_{\infty}+\|D x\|_{\infty}$, where the differential operator $D$ is given by $u=D x, \Leftrightarrow x(t)=\alpha+\int_{a}^{t} u(s) \mathrm{d} s$ and $\alpha$ is a given boundary value. Thus, $D=\frac{\mathrm{d}}{\mathrm{d} t}$ except at discontinuities.

No notational distinction is made between row and column vectors. Subscripts $x$ and $\dot{x}$ denote partial derivatives with respect to second and third component respectively, of the function involved.

Consider the determination of piecewise smooth extremal $x=x(t), t \in I$ for the following Variational Problem (P):

(P) Minimize $\int_{a}^{b} f(t, x, \dot{x}) \mathrm{d} t$

Subject to

$$
\begin{aligned}
& x(a)=\alpha, x(b)=\beta \\
& g(t, x, \dot{x}) \geq 0, \quad t \in I
\end{aligned}
$$

Let $\mathrm{G}$, denote the set of feasible solutions of the variational problem (P).

The following assumptions are required for the subsequent definitions: Let there exist a function $\eta: I \times X \times X \rightarrow R^{n}$ with $\eta(t, x, x)=0$, a continuous function $\xi: I \times X \times X \rightarrow R^{n}$ and a real number $\rho$.

Definition 2.1. The functional $\int_{a}^{b} f(t, x, \dot{X}) \mathrm{d} t$ is said to be $\rho$-invex (strictly $\rho$-invex) of order $m(m>1)$ at $\bar{X}$ with respect to $\eta$ and $\xi$, if for all $x \in X$

$$
\int_{a}^{b} f(t, x, \dot{x}) \mathrm{d} t \geq(>) \int_{a}^{b} f(t, \bar{x}, \dot{\bar{x}}) \mathrm{d} t+\int_{a}^{b}\left[\eta(t, x, \bar{x}) f_{x}(t, \bar{x}, \dot{\bar{x}})+\frac{\mathrm{d}}{\mathrm{d} t} \eta(t, x, \bar{x}) f_{\dot{x}}(t, \bar{x}, \dot{\bar{x}})+\rho\|\xi(t, x, \bar{x})\|^{m}\right] \mathrm{d} t .
$$

Definition 2.2. The functional $\int_{a}^{b} f(t, x, \dot{x}) \mathrm{d} t$ is said to be $\rho$-invex (strictly $\rho$-invex) at $\bar{x}$ with respect to $\eta$ and $\xi$, if for all $x \in X$

$$
\int_{a}^{b} f(t, x, \dot{x}) \mathrm{d} t \geq(>) \int_{a}^{b} f(t, \bar{x}, \dot{\bar{x}}) \mathrm{d} t+\int_{a}^{b}\left[\eta(t, x, \bar{x}) f_{x}(t, \bar{x}, \dot{\bar{x}})+\frac{\mathrm{d}}{\mathrm{d} t} \eta(t, x, \bar{x}) f_{\dot{x}}(t, \bar{x}, \dot{\bar{x}})+\rho\|\xi(t, x, \bar{x})\|^{2}\right] \mathrm{d} t .
$$


That is the functional $\int_{a}^{b} f(t, x, \dot{x}) \mathrm{d} t$ is $\rho$-invex (strictly $\rho$-invex) of order 2 .

Remark 2.1.

(1) If $\xi(t, x, \bar{x})=0$, the definition 2.1 reduces to the classical definition of invexity.

(2) If $\xi(t, x, \bar{x})=\eta(t, x, \bar{x})=x-\bar{x}$, the definition 2.1 reduces to the definition of $\rho$-convexity of order $m$.

(3) Definition of invexity of order $m$ given by Bhatia and Sahay [11] is a particular case of definition 2.1.

(4) " $\rho$-invex functional" defined by Bhatia and Kumar [7] restricts the value of " $t$ ", whereas in the present definition " $t$ " is released from this restriction and hence makes it more viable.

Definition 2.3. The functional $\int_{a}^{b} f(t, x, \dot{x}) \mathrm{d} t$ is said to be $\rho$-pseudo invex (strictly $\rho$-pseudo invex) type I of order $\mathrm{m}(m>1)$ at $\bar{x}$ with respect to $\eta$ and $\xi$, if for all $x \in X$

$$
\begin{aligned}
& \int_{a}^{b}\left[\eta(t, x, \bar{x}) f_{x}(t, \bar{x}, \dot{\bar{x}})+\frac{\mathrm{d}}{\mathrm{d} t} \eta(t, x, \bar{x}) f_{\dot{x}}(t, \bar{x}, \dot{\bar{x}})\right] \mathrm{d} t \geq 0 \\
& \Rightarrow \int_{a}^{b} f(t, x, \dot{x}) \mathrm{d} t \geq(>) \int_{a}^{b}\left[f(t, \bar{x}, \dot{\bar{x}})+\rho\|\xi(t, x, \bar{x})\|^{m}\right] \mathrm{d} t
\end{aligned}
$$

Definition 2.4. The functional $\int_{a}^{b} f(t, x, \dot{x}) \mathrm{d} t$ is said to be $\rho$-quasi invex (strictly $\rho$-quasi invex) type I of order $m(m>1)$ at $\bar{x}$ with respect to $\eta$ and $\xi$, if for all $x \in X$

$$
\begin{aligned}
& \int_{a}^{b} f(t, x, \dot{x}) \mathrm{d} t \leq \int_{a}^{b}\left[f(t, \bar{x}, \dot{\bar{x}})+\rho\|\xi(t, x, \bar{x})\|^{m}\right] \mathrm{d} t \\
& \Rightarrow \int_{a}^{b}\left[\eta(t, x, \bar{x}) f_{x}(t, \bar{x}, \dot{\bar{x}})+\frac{\mathrm{d}}{\mathrm{d} t} \eta(t, x, \bar{x}) f_{\dot{x}}(t, \bar{x}, \dot{\bar{x}})\right] \mathrm{d} t \leq(<) 0
\end{aligned}
$$

Definition 2.5. The functional $\int_{a}^{b} f(t, x, \dot{x}) \mathrm{d} t$ is said to be $\rho$-pseudo invex (strictly $\rho$-pseudo invex) type II of order $m(m>1)$ at $\bar{x}$ with respect to $\eta$ and $\xi$, if for all $x \in X$

$$
\begin{aligned}
& \int_{a}^{b}\left[\eta(t, x, \bar{x}) f_{x}(t, \bar{x}, \dot{\bar{x}})+\frac{\mathrm{d}}{\mathrm{d} t} \eta(t, x, \bar{x}) f_{\dot{x}}(t, \bar{x}, \dot{\bar{x}})+\rho\|\xi(t, x, \bar{x})\|^{m}\right] \mathrm{d} t \geq 0 \\
& \Rightarrow \int_{a}^{b} f(t, x, \dot{x}) \mathrm{d} t \geq(>) \int_{a}^{b} f(t, \bar{x}, \dot{\bar{x}}) \mathrm{d} t
\end{aligned}
$$

Definition 2.6. The functional $\int_{a}^{b} f(t, x, \dot{x}) \mathrm{d} t$ is said to be $\rho$-quasi invex (strictly $\rho$-quasi invex) type II of order $m(m>1)$ at $\bar{x}$ with respect to $\eta$ and $\xi$, if for all $x \in X$

$$
\begin{aligned}
& \int_{a}^{b} f(t, x, \dot{x}) \mathrm{d} t \leq \int_{a}^{b} f(t, \bar{x}, \dot{\bar{x}}) \mathrm{d} t \\
& \Rightarrow \int_{a}^{b}\left[\eta(t, x, \bar{x}) f_{x}(t, \bar{x}, \dot{\bar{x}})+\frac{\mathrm{d}}{\mathrm{d} t} \eta(t, x, \bar{x}) f_{\dot{x}}(t, \bar{x}, \dot{\bar{x}})+\rho\|\xi(t, x, \bar{x})\|^{m}\right] \mathrm{d} t \leq(<) 0
\end{aligned}
$$

Remark 2.2. Every $\rho$-invex functional of order $m(m>1)$ at $\bar{x}$ with respect to $\eta$ and $\xi$, is $\rho$-pseudo invex type I functional of order $m(m>1)$ as well as $\rho$-quasi invex type I functional of order $m(m>1)$ at $\bar{x}$ with respect to same $\eta$ and $\xi$.

Remark 2.3. It can be easily proved that every $\rho$-invex functional is a $\rho$-invex functional of order $m(m>1)$ with respect to same $\eta$ and $\xi$ but the converse may not be true which is shown in the following example. This illustrates the fact that the class of $\rho$-invex functionals of order $m$ is larger than the class of $\rho$-invex functionals.

Example 2.1. Let $f(t, x, \dot{x})=2 x(t) \dot{x}(t), t \in[0,1]$ and $x(0)=1, \quad x(1)=2$.

Claim: $\int_{a}^{b} f(t, x, \dot{x}) \mathrm{d} t$ is $\rho$-invex functional of order $m(m=4)$ at $\bar{x}(t)=0$, with respect to $\eta(t, x, \bar{x})=\frac{t}{\dot{\bar{x}}}$, $\xi(t, x, \bar{x})=\bar{x}+\frac{1}{2}$, and $\rho=16$, but it is not $\rho$-invex functional at $\bar{x}(t)=0$, with respect to same $\eta$ and $\xi$.

Applying the definition of $\rho$-invexity of the functional $\int_{a}^{b} f(t, x, \dot{x}) \mathrm{d} t$, we obtain

$$
\int_{0}^{1} 2 x(t) \dot{x}(t) \mathrm{d} t+\int_{0}^{1} 2 \bar{x}(t) \dot{\bar{x}}(t) \mathrm{d} t \geq \int_{0}^{1}\left[\frac{t}{\dot{\bar{x}}}(2 \dot{\bar{x}}(t))+\frac{\mathrm{d} \eta}{\mathrm{d} t} 2 \bar{x}(t)+16\left\|\bar{x}(t)+\frac{1}{2}\right\|^{2}\right] \mathrm{d} t
$$


At $\bar{x}(t)=0$, the above inequality reduces to

$$
\int_{0}^{1} 2 x \dot{x}(t) \mathrm{d} t \geq \int_{0}^{1}\left[2 t+16\left\|\frac{1}{2}\right\|^{2}\right] \mathrm{d} t=\int_{0}^{1}[2 t+4] \mathrm{d} t
$$

Integrating both sides and putting limits, we obtain $3 \geq 1+4=5$, This is not true.

Hence the functional $\int_{a}^{b} f(t, x, \dot{x}) \mathrm{d} t$ is not $\rho$-invex.

Now, the definition of $\rho$-invexity of order $m=4$ of the functional $\int_{a}^{b} f(t, x, \dot{x}) \mathrm{d} t$ yields

$$
\int_{0}^{1} 2 x \dot{x}(t) \mathrm{d} t \geq \int_{0}^{1}\left[2 t+16\left\|\frac{1}{2}\right\|^{4}\right] \mathrm{d} t=\int_{0}^{1}[2 t+1] \mathrm{d} t
$$

$3 \geq 1+16\left\|\frac{1}{2}\right\|^{4}=1+1=2$, and this is true.

Hence the functional $\int_{a}^{b} f(t, x, \dot{x}) \mathrm{d} t$ is $\rho$-invex of order $m=4$.

In fact the functional $\int_{a}^{b} f(t, x, \dot{x}) \mathrm{d} t$ is $\rho$-invex of order $m$ for any $m \geq 4$.

\section{Sufficient Optimality Conditions}

In this section, we establish various sufficient optimality conditions for the variational problem (P) using higher order $\rho$-invexity assumptions (for notational convenience, we write $y$ in place of $y(t)$ ).

Theorem 3.1. Let $\bar{x}$ be feasible for (P) and let there exists piecewise smooth function $y: I \rightarrow R^{p}$ such that, for all $t \in I$

$$
\begin{aligned}
& f_{x}(t, \bar{x}, \dot{\bar{x}})-y(t)^{\mathrm{T}} g_{x}(t, \bar{x}, \dot{\bar{x}})=0 \\
& f_{\dot{x}}(t, \bar{x}, \dot{\bar{x}})-y(t)^{\mathrm{T}} g_{\dot{x}}(t, \bar{x}, \dot{\bar{x}})=0 \\
& y(t)^{\mathrm{T}} g(t, \bar{x}, \dot{\bar{x}})=0 \\
& y(t) \geq 0
\end{aligned}
$$

Let either of the following conditions hold

(a) $\int_{a}^{b} f(t, x, \dot{x}) \mathrm{d} t$ is $\rho$-invex functional of order $m$ at $\bar{x}$ with respect to $\eta$ and $\xi$ and $-\int_{a}^{b} y_{i} g_{i}(t, x, \dot{x}) \mathrm{d} t$ is $\rho_{i}^{\prime}$-invex functional of order $m$ for all $i=1,2, \cdots, p$, at $\bar{x}$ with respect to same $\eta$ and $\xi$, where

$$
\left(\rho+\sum_{i=1}^{p} \rho^{\prime}\right) \geq 0
$$

(b) $\int_{a}^{b} f(t, x, \dot{x}) \mathrm{d} t$ is $\rho$-invex functional of order $m$ at $\bar{x}$ with respect to $\eta$ and $\xi$ and $-\int_{a}^{b} y^{\mathrm{T}} g(t, x, \dot{x}) \mathrm{d} t$ is $\rho^{\prime}$-invex functional of order $m$ at $\bar{x}$ with respect to same $\eta$ and $\xi$ where, $\left(\rho+\rho^{\prime}\right) \geq 0$,

Then $\bar{x}$ is an optimal solution for $(\mathrm{P})$.

Proof (a). Let $x$ be any feasible solution of (P). Since $-\int_{a}^{b} y_{i} g_{i}(t, x, \dot{x}) \mathrm{d} t$ is $\rho_{i}^{\prime}$-invex functional of order $m$ at $\bar{x}$ with respect to $\eta$ and $\xi$,

$$
\begin{aligned}
& -\int_{a}^{b} y_{i} g_{i}(t, x, \dot{x}) \mathrm{d} t-\left\{-\int_{a}^{b} y_{i} g_{i}(t, \bar{x}, \dot{\bar{x}}) \mathrm{d} t\right\} \\
& \geq-\int_{a}^{b}\left[\eta(t, x, \bar{x}) y_{i} g_{i x}(t, \bar{x}, \dot{\bar{x}})-\frac{\mathrm{d}}{\mathrm{d} t} \eta(t, x, \dot{x}) y_{i} g_{i \dot{x}}(t, \bar{x}, \dot{\bar{x}})+\rho_{i}^{\prime}\|\xi(t, x, \bar{x})\|^{m}\right] \mathrm{d} t \text {, for all } i=1,2, \cdots, p
\end{aligned}
$$

Multiplying each of the above inequality by -1 and taking summation over $i$ yields

$$
\begin{aligned}
& \int_{a}^{b} y^{\mathrm{T}} g(t, x, \dot{x}) \mathrm{d} t-\int_{a}^{b} y^{\mathrm{T}} g(t, \bar{x}, \dot{\bar{x}}) \mathrm{d} t \\
& \leq \int_{a}^{b}\left[\eta(t, x, \bar{x}) y^{\mathrm{T}} g_{x}(t, \bar{x}, \dot{\bar{x}})+\frac{\mathrm{d}}{\mathrm{d} t} \eta(t, x, \bar{x}) y^{\mathrm{T}} g_{\dot{x}}(t, \bar{x}, \dot{\bar{x}})-\sum_{i=1}^{p} \rho_{i}^{\prime}\|\xi(t, x, \bar{x})\|^{m}\right] \mathrm{d} t
\end{aligned}
$$


Since $\int_{a}^{b} f(t, x, \dot{x}) \mathrm{d} t$ is $\rho$-invex functional of order $m$ at $\bar{x} \quad$ w.r.t. to $\eta$ and $\xi$, therefore for $x \in G$,

$$
\int_{a}^{b} f(t, x, \dot{x}) \mathrm{d} t-\int_{a}^{b} f(t, \bar{x}, \dot{\bar{x}}) \mathrm{d} t \geq \int_{a}^{b}\left[\eta(t, x, \bar{x}) f_{x}(t, \bar{x}, \dot{\bar{x}})+\frac{\mathrm{d}}{\mathrm{d} t} \eta(t, x, \bar{x}) f_{\dot{x}}(t, \bar{x}, \dot{\bar{x}})+\rho\|\xi(t, x, \bar{x})\|^{m}\right] \mathrm{d} t
$$

Equations (3) and (4) along with the above inequality yields

$$
\begin{aligned}
& \int_{a}^{b} f(t, x, \dot{x}) \mathrm{d} t-\int_{a}^{b} f(t, \bar{x}, \dot{\bar{x}}) \mathrm{d} t \\
& \geq \int_{a}^{b}\left[\eta(t, x, \bar{x}) y^{\mathrm{T}} g_{x}(t, \bar{x}, \dot{\bar{x}})+\frac{\mathrm{d}}{\mathrm{d} t} \eta(t, x, \bar{x}) y^{\mathrm{T}} g_{\dot{x}}(t, \bar{x}, \dot{\bar{x}})+\rho\|\xi(t, x, \bar{x})\|^{m}\right] \mathrm{d} t \\
& =\int_{a}^{b}\left[y^{\mathrm{T}}\left\{\eta(t, x, \bar{x}) g_{x}(t, \bar{x}, \dot{\bar{x}})+\frac{\mathrm{d}}{\mathrm{d} t} \eta(t, x, \bar{x}) g_{\dot{x}}(t, \bar{x}, \dot{\bar{x}})\right\}+\rho\|\xi(t, x, \bar{x})\|^{m}\right] \mathrm{d} t \\
& \geq \int_{a}^{b}\left[\left\{y^{\mathrm{T}} g(t, x, \dot{x})-\int_{a}^{b} y^{\mathrm{T}} g(t, \bar{x}, \dot{\bar{x}})\right\}+\rho\|\xi(t, x, \bar{x})\|^{m}+\sum_{i=1}^{p} \rho_{i}^{\prime}\|\xi(t, x, \bar{x})\|^{m}\right] \mathrm{d} t \text { (by using (8)) } \\
& =\int_{a}^{b} y^{\mathrm{T}} g(t, x, \dot{x}) \mathrm{d} t-\int_{a}^{b} y^{\mathrm{T}} g(t, \bar{x}, \dot{\bar{x}}) \mathrm{d} t+\int_{a}^{b}\left(\rho+\sum_{i=1}^{p} \rho_{i}^{\prime}\right)\|\xi(t, x, \bar{x})\|^{m} \mathrm{~d} t \\
& \geq 0(\text { by using equations }(5),(6) \text { and }(7)) \\
& \Rightarrow \int_{a}^{b} f(t, x, \dot{x}) \mathrm{d} t \geq \int_{a}^{b} f(t, \bar{x}, \dot{\bar{x}}) \mathrm{d} t .
\end{aligned}
$$

Hence $\bar{x}$ is an optimal solution for (P).

(b). Proof of this part follows on the lines of part (a) and is hence omitted.

Theorem 3.2. Let $\bar{x}$ be feasible for (P) and let there exists piece wise smooth function $y: I \rightarrow R^{p}$ such that for all $t \in I$, conditions (3)-(6) are satisfied. Let either of the following conditions hold

(a) $\int_{a}^{b} f(t, x, \dot{x}) \mathrm{d} t$ is $\rho$-pseudo-invex type II functional of order $m$ at $\bar{x}$ with respect to $\eta$ and $\xi$, where $\rho \geq 0$ and $-\int_{a}^{b} y_{i} g_{i}(t, x, \dot{x}) \mathrm{d} t$ are $\rho_{i}^{\prime}$-quasi-invex type II functional of order $m$, for all $i=1,2, \cdots, p$ at $\bar{x}$ with respect to same $\eta$ and $\xi$ where $\sum_{i=1}^{p} \rho_{i}^{\prime} \geq 0$.

(b) $\int_{a}^{b} f(t, x, \dot{x}) \mathrm{d} t$ is $\rho$-pseudo-invex type II functional of order $m$ at $\bar{x}$ with respect to $\eta$ and $\xi, \rho \geq 0$ and $-\int_{a}^{b} y^{\mathrm{T}} g(t, x, \dot{x}) \mathrm{d} t$ is $\rho_{i}^{\prime}$-quasi-invex type II functional of order $m$ at $\bar{x}$ with respect to same $\eta$ and $\xi$, where $\rho_{i}^{\prime} \geq 0$, for all $i=1,2, \cdots, p$.

Then $\bar{x}$ is an optimal solution for $(\mathrm{P})$.

Proof (a). Let $x$ be any feasible solution of (P). Feasibility of $x$ along with Equation (6) implies $\int_{a}^{b} y_{i} g_{i}(t, x, \dot{x}) \mathrm{d} t \geq 0$.

Since $\bar{x}$ satisfies Equation (5), we get $\int_{a}^{b} y_{i} g_{i}(t, x, \dot{x}) \mathrm{d} t \geq \int_{a}^{b} y_{i} g_{i}(t, \bar{x}, \dot{\bar{x}}) \mathrm{d} t$, for all $i=1,2, \cdots, p$, which implies $-\int_{a}^{b} y_{i} g_{i}(t, x, \dot{x}) \mathrm{d} t \leq-\int_{a}^{b} y_{i} g_{i}(t, \bar{x}, \dot{\bar{x}}) \mathrm{d} t$, for all $i=1,2, \cdots, p$.

Since $-\int_{a}^{b} y_{i} g_{i}(t, x, \dot{x}) \mathrm{d} t$ is $\rho_{i}^{\prime}$-quasi-invex type II functional of order $m$ at $\bar{x}$ with respect to $\eta$ and $\xi$, for all $i=1,2, \cdots, p$

$$
\int_{a}^{b}\left[-\eta(t, x, \bar{x}) y_{i} g_{i x}(t, \bar{x}, \dot{\bar{x}})-\frac{\mathrm{d}}{\mathrm{d} t} \eta(t, x, \bar{x}) y_{i} g_{i \dot{x}}(t, \bar{x}, \dot{\bar{x}})+\rho_{i}^{\prime}\|\xi(t, x, \bar{x})\|^{m}\right] \mathrm{d} t \leq 0, \text { for all } i=1,2, \cdots, p
$$

Multiplying each of the above inequality by -1 and taking summation over $i$ yields

$$
\begin{aligned}
& \int_{a}^{b}\left[\eta(t, x, \bar{x}) y^{\mathrm{T}} g_{x}(t, \bar{x}, \dot{\bar{x}})+\frac{\mathrm{d}}{\mathrm{d} t} \eta(t, x, \bar{x}) y^{\mathrm{T}} g_{\dot{x}}(t, \bar{x}, \dot{\bar{x}})-\sum_{i=1}^{p} \rho_{i}^{\prime}\|\xi(t, x, \bar{x})\|^{m}\right] \mathrm{d} t \geq 0 \\
& \Rightarrow \int_{a}^{b}\left[\eta(t, x, \bar{x}) y^{\mathrm{T}} g_{x}(t, \bar{x}, \dot{\bar{x}})+\frac{\mathrm{d}}{\mathrm{d} t} \eta(t, x, \bar{x}) y^{\mathrm{T}} g_{\dot{x}}(t, \bar{x}, \dot{\bar{x}})\right] \mathrm{d} t \geq 0
\end{aligned}
$$

Using Equations (3) and (4) in (9) we get

$$
\int_{a}^{b}\left[\eta(t, x, \bar{x}) f_{x}(t, \bar{x}, \dot{\bar{x}})+\frac{\mathrm{d}}{\mathrm{d} t} \eta(t, x, \bar{x}) f_{\dot{x}}(t, \bar{x}, \dot{\bar{x}})\right] \mathrm{d} t \geq 0
$$




$$
\Rightarrow \int_{a}^{b}\left[\eta(t, x, \bar{x}) f_{x}(t, \bar{x}, \dot{\bar{x}})+\frac{\mathrm{d}}{\mathrm{d} t} \eta(t, x, \bar{x}) f_{\dot{x}}(t, \bar{x}, \dot{\bar{x}})+\rho\|\xi(t, x, \bar{x})\|^{m}\right] \mathrm{d} t \geq 0
$$

Since $\int_{a}^{b} f(t, x, \dot{x}) \mathrm{d} t$ is strictly $\rho$-pseudo-invex type II functional of order $m$ at $\bar{x}$ with respect to $\eta$ and $\xi$, therefore

$$
\int_{a}^{b} f(t, x, \dot{x}) \mathrm{d} t>\int_{a}^{b} f(t, \bar{x}, \dot{\bar{x}}) \mathrm{d} t
$$

Hence $\bar{X}$ is an optimal solution for $(\mathrm{P})$.

(b). Proof of this part follows on the same lines as that of part (a) and is hence omitted.

\section{Duality}

\subsection{The Wolfe Dual}

The Wolfe type dual problem (WD) associated with $(\mathrm{P})$ is given by

(WD) Maximize $\int_{a}^{b}[f(t, u, \dot{u})-y(t) g(t, u, \dot{u})] \mathrm{d} t$

Subject to

$$
\begin{gathered}
u(a)=\alpha, u(b)=\beta \\
f_{x}(t, u, \dot{u})-y(t) g_{x}(t, u, \dot{u})=\frac{\mathrm{d}}{\mathrm{d} t}\left[f_{\dot{x}}(t, u, \dot{u})-y(t) g_{\dot{x}}(t, u, \dot{u})\right] \\
y(t) \geq 0
\end{gathered}
$$

Here, $y: I \rightarrow R^{p}$ is continuous except possibly for the values of $t$ corresponding to the corners of $x(t)$. For such values of $t$, Equation (11) must be satisfied for right and left hand limits.

We now establish duality results between (P) and (WD) using higher order generalized $\rho$-invexity assumptions.

Theorem 4.1. (Weak Duality) Let $x$ be a feasible solution for (P) and $(u, y)$ be a feasible solution for (WD). If the functional $\int_{a}^{b}(f-y g) \mathrm{d} t$ is $\rho$-pseudo invex type I of order $m$ at $u$ with respect to $\eta$ and $\xi$ where $\rho>0$, then

$$
\int_{a}^{b} f(t, x, \dot{x}) \mathrm{d} t \geq \int_{a}^{b}[f(t, u, \dot{u})-y(t) g(t, u, \dot{u})] \mathrm{d} t .
$$

Proof. Since $x$ is a feasible solution for (P) and $(u, y)$ is a feasible solution for (WD), from (11) it follows that

$$
\int_{a}^{b}\left[\eta(t, x, u)\left(f_{x}(t, u, \dot{u})-y(t) g_{x}(t, u, \dot{u})\right)-\eta(t, x, u) \frac{\mathrm{d}}{\mathrm{d} t}\left(f_{\dot{x}}(t, u, \dot{u})-y(t) g_{\dot{x}}(t, u, \dot{u})\right)\right] \mathrm{d} t=0
$$

Now integrating by parts the following function and applying boundary conditions (1) and (10), we get

$$
\int_{a}^{b}\left[\frac{\mathrm{d}}{\mathrm{d} t} \eta(t, x, u)\left(f_{\dot{x}}(t, u, \dot{u})-y(t) g_{\dot{x}}(t, u, \dot{u})\right)\right] \mathrm{d} t=-\int_{a}^{b} \eta(t, x, u) \frac{\mathrm{d}}{\mathrm{d} t}\left[f_{\dot{x}}(t, u, \dot{u})-y(t) g_{\dot{x}}(t, u, \dot{u})\right] \mathrm{d} t
$$

It follows from (13) and (14) that

$$
\int_{a}^{b}\left[\eta(t, x, u)\left(f_{x}(t, u, \dot{u})-y(t) g_{x}(t, u, \dot{u})\right)+\frac{\mathrm{d}}{\mathrm{d} t} \eta(t, x, u)\left(f_{\dot{x}}(t, u, \dot{u})-y(t) g_{\dot{x}}(t, u, \dot{u})\right)\right] \mathrm{d} t=0
$$

Since the functional $\int_{a}^{b}(f-y g) \mathrm{d} t$ is $\rho$-pseudo invex type I of order $m$ at $u$ with respect to $\eta$ and $\xi$, the above equation yields

$$
\int_{a}^{b}[f(t, x, \dot{x})-y(t) g(t, x, \dot{x})] \mathrm{d} t \geq \int_{a}^{b}\left[f(t, u, \dot{u})-y(t) g(t, u, \dot{u})+\rho\|\xi(t, x, \bar{x})\|^{m}\right] \mathrm{d} t
$$


Now, contrary to the result, assume that

$$
\int_{a}^{b} f(t, x, \dot{x}) \mathrm{d} t<\int_{a}^{b}[f(t, u, \dot{u})-y(t) g(t, u, \dot{u})] \mathrm{d} t
$$

Using Equations (2) and (12) we get

$$
\int_{a}^{b}[f(t, x, \dot{x})-y(t) g(t, x, \dot{x})] \mathrm{d} t<\int_{a}^{b}[f(t, u, \dot{u})-y(t) g(t, u, \dot{u})] \mathrm{d} t
$$

Since $\rho>0$ the above inequality yields

$$
\int_{a}^{b}[f(t, x, \dot{x})-y(t) g(t, x, \dot{x})] \mathrm{d} t<\int_{a}^{b}\left[f(t, u, \dot{u})-y(t) g(t, u, \dot{u})+\rho\|\xi(t, x, \bar{x})\|^{m}\right] \mathrm{d} t
$$

But this contradicts (15). Hence the result follows.

The following theorem by Mond and Hanson is required to prove the strong duality theorem.

Theorem 4.2. (Mond and Hanson [4]) For every minimizing arc $x=x^{*}(t)$ of (P) there exists a function of the form

$$
G=y_{\circ}^{*} f(t, x, \dot{x})-y^{*}(t) g(t, x, \dot{x})
$$

such that

$$
\begin{gathered}
G_{x}=\frac{\mathrm{d}}{\mathrm{d} t} G_{\dot{x}} \\
y^{*}(t) g(t, x, \dot{x})=0 \\
y^{*}(t) \geq 0
\end{gathered}
$$

holds throughout I (except at the corners of $x^{*}(t)$, where (16) holds for unique right and left hand limits). Here $y_{\circ}^{*}$ is constant and $y$ is continuous except possibly for values of $t$ corresponding to corners of $x^{*}(t)$. Further $\left(y_{\circ}^{*}, y(t)\right)$ cannot vanish for any $t \in I$. If $x^{*}(t)$ is normal, $y_{\circ}^{*}$ can be taken equal to 1 .

Theorem 4.3. (Strong Duality) Let $x^{*}(t)$ minimizes the primal problem (P) and assume that $x^{*}(t)$ is normal, then there exists $y^{*}(t)$ such that $\left(x^{*}(t), y^{*}(t)\right)$ is feasible for (WD). Further if conditions of weak duality (Theorem 4.1) are satisfied then $\left(x^{*}, y^{*}\right)$ maximizes (WD) and extreme values of (P) and (WD) are equal.

Proof. Since $x^{*}$ minimizes $(\mathrm{P})$ and is normal, it follows from theorem 4.2, that there exists $y^{*}: I \rightarrow R^{p}$ satisfying

$$
\begin{gathered}
f_{x}\left(t, x^{*}, \dot{x}^{*}\right)-y^{*}(t) g_{x}\left(t, x^{*}, \dot{x}^{*}\right)=\frac{\mathrm{d}}{\mathrm{d} t}\left[f_{\dot{x}}\left(t, x^{*}, \dot{x}^{*}\right)-y^{*}(t) g_{\dot{x}}\left(t, x^{*}, \dot{x}^{*}\right)\right] \\
y^{*}(t) g\left(t, x^{*}, \dot{x}^{*}\right)=0 \\
y^{*}(t) \geq 0
\end{gathered}
$$

It follows from (19) and (21) that $\left(x^{*}, y^{*}\right)$ is feasible for (WD). Result now follows directly from Equation (20) and weak duality theorem (4.1).

Theorem 4.4. (Strict Converse Duality) Let $\bar{x}$ be an optimal solution of $(\mathrm{P})$ and $(\bar{u}, \bar{y})$ be an optimal solution of (WD) such that

$$
\int_{a}^{b} f(t, \bar{x}, \dot{\bar{x}}) \mathrm{d} t=\int_{a}^{b}[f(t, \bar{u}, \dot{\bar{u}})-\bar{y}(t) g(t, \bar{u}, \dot{\bar{u}})] \mathrm{d} t
$$

Assume that the functional $\int_{a}^{b}(f-\bar{y} g) \mathrm{d} t$ is strictly $\rho$-pseudo invex type I of order $m$ on $X$ with respect to $\eta$ and $\xi$, where $\rho>0$. Then $\bar{x}=\bar{u}$, and $\bar{u}$ is an optimal solution of (P).

Proof. Since $\int_{a}^{b} f(t, \bar{x}, \dot{\bar{x}}) \mathrm{d} t=\int_{a}^{b}[f(t, \bar{u}, \dot{\bar{u}})-\bar{y}(t) g(t, \bar{u}, \dot{\bar{u}})] \mathrm{d} t$ 
It follows from Equations (2) and (12) that

$$
\begin{aligned}
& \int_{a}^{b}[f(t, \bar{x}, \dot{\bar{x}})-\bar{y}(t) g(t, \bar{x}, \dot{\bar{x}})] \mathrm{d} t \leq \int_{a}^{b}[f(t, \bar{u}, \dot{\bar{u}})-\bar{y}(t) g(t, \bar{u}, \dot{\bar{u}})] \mathrm{d} t \\
& \Rightarrow \int_{a}^{b}[f(t, \bar{x}, \dot{\bar{x}})-\bar{y}(t) g(t, \bar{x}, \dot{\bar{x}})] \mathrm{d} t \leq \int_{a}^{b}\left[f(t, \bar{u}, \dot{\bar{u}})-\bar{y}(t) g(t, \bar{u}, \dot{\bar{u}})+\rho\|\xi(t, x, u)\|^{m}\right] \mathrm{d} t
\end{aligned}
$$

Now, if $\bar{x} \neq \bar{u}$, it follows from Equation (11) that

$$
\int_{a}^{b}\left[\eta(t, \bar{x}, \bar{u})\left\{f_{x}(t, \bar{u}, \dot{\bar{u}})-\bar{y}(t) g_{x}(t, \bar{u}, \dot{\bar{u}})\right\}-\eta(t, \bar{x}, \bar{u}) \frac{\mathrm{d}}{\mathrm{d} t}\left\{f_{\dot{x}}(t, \bar{u}, \dot{\bar{u}})-\bar{y}(t) g_{\dot{x}}(t, \bar{u}, \dot{\bar{u}})\right\}\right] \mathrm{d} t=0
$$

Using Equation (14), we get

$$
\int_{a}^{b}\left[\eta(t, \bar{x}, \bar{u})\left\{f_{x}(t, \bar{u}, \dot{\bar{u}})-\bar{y}(t) g_{x}(t, \bar{u}, \dot{\bar{u}})\right\}+\frac{\mathrm{d}}{\mathrm{d} t} \eta(t, \bar{x}, \bar{u})\left\{f_{\dot{x}}(t, \bar{u}, \dot{\bar{u}})-\bar{y}(t) g_{\dot{x}}(t, \bar{u}, \dot{\bar{u}})\right\}\right] \mathrm{d} t=0
$$

Since $\int_{a}^{b}(f-\bar{y} g) \mathrm{d} t$ is strict $\rho$-pseudo invex type I functional of order $m$ with respect to $\eta$ and $\xi$, therefore the above equation yields

$$
\int_{a}^{b}[f(t, \bar{x}, \dot{\bar{x}})-\bar{y}(t) g(t, \bar{x}, \dot{\bar{x}})] \mathrm{d} t>\int_{a}^{b}\left[f(t, \bar{u}, \dot{\bar{u}})-\bar{y}(t) g(t, \bar{u}, \dot{\bar{u}})+\rho\|\xi(t, x, u)\|^{m}\right] \mathrm{d} t
$$

This contradicts Equation (22). Hence $\bar{x}=\bar{u}$ implying there by that $\bar{u}$ is an optimal solution of (P).

\subsection{Mond-Weir Dual}

The Mond-Weir dual problem (MD) associated with (P) is given by

(MD) Maximize $\int_{a}^{b} f(t, u, \dot{u}) \mathrm{d} t$

Subject to

$$
\begin{gathered}
u(a)=\alpha, u(b)=\beta \\
f_{x}(t, u, \dot{u})-y(t) g_{x}(t, u, \dot{u})=\frac{\mathrm{d}}{\mathrm{d} t}\left[f_{\dot{x}}(t, u, \dot{u})-y(t) g_{\dot{x}}(t, u, \dot{u})\right] \\
y(t) g(t, u, \dot{u}) \leq 0 \\
y(t) \geq 0
\end{gathered}
$$

Here, $y: I \rightarrow R^{p}$ is continuous except possibly for the values of $t$ corresponding to the corners of $x(t)$. For such values of $t$, Equation (24) must be satisfied for right and left hand limits.

Theorem 4.5. (Weak Duality) Let $x$ be a feasible solution for (P) and $(u, y)$ be a feasible solution for (MD), if $\int_{a}^{b} f(t, u, \dot{u}) \mathrm{d} t$ is $\rho$-pseudo invex type I functional of order $m$ at $u$ with respect to $\eta$ and $\xi$ where $\rho>0$ and $-\int_{a}^{b} y(t) g(t, x, \dot{x}) \mathrm{d} t$ is $\rho^{\prime}$-quasi invex type I functional of order $m$ at $u$ with respect to the same $\eta$ and $\xi$ where $\rho^{\prime}>0$, then $\int_{a}^{b} f(t, x, \dot{x}) \mathrm{d} t \geq \int_{a}^{b} f(t, u, \dot{u}) \mathrm{d} t$.

Proof. Since $x$ is feasible solution for (P) and $(u, y)$ is feasible solution for (MD), it follows from (2), (12) and (25) that

$$
\int_{a}^{b}[y(t) g(t, x, \dot{x})] \mathrm{d} t \geq \int_{a}^{b}[y(t) g(t, u, \dot{u})] \mathrm{d} t
$$

Now $\rho^{\prime}>0$ implies that

$$
-\int_{a}^{b} y(t) g(t, x, \dot{x}) \mathrm{d} t \leq-\int_{a}^{b} y(t) g(t, u, \dot{u}) \mathrm{d} t+\int_{a}^{b} \rho^{\prime}\|\xi(t, x, u)\|^{m} \mathrm{~d} t
$$

As $-\int_{a}^{b} y(t) g(t, x, \dot{x}) \mathrm{d} t$ is $\rho^{\prime}$-quasi invex type I functional of order $\mathrm{m}$ with respect to $\eta$ and $\xi$, above inequality yields 


$$
-\int_{a}^{b}\left[\eta(t, x, u) y(t) g_{x}(t, u, \dot{u})+\frac{\mathrm{d}}{\mathrm{d} t} \eta(t, x, u) y(t) g_{\dot{x}}(t, u, \dot{u})\right] \mathrm{d} t \leq 0
$$

Integrating by parts the followings functions and using boundary conditions (1) and (23), we get

$$
\int_{a}^{b} \frac{\mathrm{d}}{\mathrm{d} t} \eta(t, x, u) y(t) g_{\dot{x}}(t, u, \dot{u}) \mathrm{d} t=-\int_{a}^{b} \eta(t, x, u) \frac{\mathrm{d}}{\mathrm{d} t}\left[y(t) g_{\dot{x}}(t, u, \dot{u})\right] \mathrm{d} t
$$

Using (28) in (27)

$$
\int_{a}^{b} \eta(t, x, u)\left[-y(t) g_{x}(t, u, \dot{u})+\frac{\mathrm{d}}{\mathrm{d} t} y(t) g_{\dot{x}}(t, u, \dot{u})\right] \mathrm{d} t \leq 0
$$

Now, dual feasibility constraint (24) yields

$$
\begin{aligned}
& \int_{a}^{b} \eta(t, x, u)\left[-f_{x}(t, u, \dot{u})+\frac{\mathrm{d}}{\mathrm{d} t} f_{\dot{x}}(t, u, \dot{u})\right] \mathrm{d} t \leq 0 \\
& \Rightarrow \int_{a}^{b}\left[\eta(t, x, u) f_{x}(t, u, \dot{u})-\eta(t, x, u) \frac{\mathrm{d}}{\mathrm{d} t} f_{\dot{x}}(t, u, \dot{u})\right] \mathrm{d} t \geq 0
\end{aligned}
$$

Again, integrating by parts the followings functions and using boundary conditions (1) and (23), we get

$$
\int_{a}^{b}\left[\eta(t, x, u) \frac{\mathrm{d}}{\mathrm{d} t} f_{\dot{x}}(t, u, \dot{u})\right] \mathrm{d} t=-\int_{a}^{b}\left[\frac{\mathrm{d}}{\mathrm{d} t} \eta(t, x, u) f_{\dot{x}}(t, u, \dot{u})\right] \mathrm{d} t
$$

Equations (29) and (30) yield

$$
\int_{a}^{b}\left[\eta(t, x, u) f_{x}(t, u, \dot{u})+\frac{\mathrm{d}}{\mathrm{d} t} \eta(t, x, u) f_{\dot{x}}(t, u, \dot{u})\right] \mathrm{d} t \geq 0
$$

As $\int_{a}^{b} f(t, x, \dot{x}) \mathrm{d} t$ is $\rho$-pseudo invex type I functional of order $m$ with respect to $\eta$ and $\xi$, the above inequality yields

$$
\int_{a}^{b} f(t, x, \dot{x}) \mathrm{d} t \geq \int_{a}^{b}\left[f(t, u, \dot{u})+\rho\|\xi(t, x, u)\|^{m}\right] \mathrm{d} t
$$

Since $\rho \geq 0$ the above inequality yields

$$
\int_{a}^{b} f(t, x, \dot{x}) \mathrm{d} t \geq \int_{a}^{b} f(t, u, \dot{u}) \mathrm{d} t
$$

Hence the result follows.

Theorem 4.6. (Weak Duality) Let $x$ be a feasible solution for (P) and $(u, y)$ be a feasible solution for (MD), if $\int_{a}^{b}(f-y g) \mathrm{d} t$ is strictly $\rho$-pseudo invex type I functional of order $m$ at $u$ with respect to $\eta$ and $\xi$, where $\rho>0$, then

$$
\int_{a}^{b} f(t, x, \dot{x}) \mathrm{d} t \geq \int_{a}^{b} f(t, u, \dot{u}) \mathrm{d} t .
$$

Proof. Proceeding on the similar lines as in theorem 4.1, we arrive at the following inequality

$$
\int_{a}^{b} f(t, x, \dot{x}) \mathrm{d} t \geq \int_{a}^{b}[f(t, u, \dot{u})-y(t) g(t, u, \dot{u})] \mathrm{d} t
$$

where $x$ is feasible for $(\mathrm{P})$ and $(u, y)$ is feasible for (MD).

Now, using the dual feasibility condition (25) we get

$$
\int_{a}^{b} f(t, x, \dot{x}) \mathrm{d} t \geq \int_{a}^{b} f(t, u, \dot{u}) \mathrm{d} t,
$$

this proves the theorem. 
Theorem 4.7. (Strong Duality) Assume that $x^{*}(t)$ is optimal for (P) and is normal, then there exists $y^{*}(t)$ such that $\left(x^{*}, y^{*}\right)$ is feasible for (MD). Further if conditions of weak duality (Theorem 4.5 or 4.6) are satisfied, then $\left(x^{*}, y^{*}\right)$ is optimal for (MD) and extreme value of (P) and (MD) are equal.

Proof: Proof runs on the similar lines as that of theorem 4.3 and is hence omitted.

Theorem 4.8. (Strict Converse Duality) Let $\bar{x}$ be optimal for (P) and $(\bar{u}, \bar{y})$ be optimal for (MD) such that

$$
\int_{a}^{b} f(t, \bar{x}, \dot{\bar{x}}) \mathrm{d} t=\int_{a}^{b} f(t, \bar{u}, \dot{\bar{u}}) \mathrm{d} t .
$$

If $\int_{a}^{b}(f-y g) \mathrm{d} t$ is strictly $\rho$-pseudo invex type I functional of order $m$ with respect to $\eta$ and $\xi$, then $\bar{x}=\bar{u}$, and $\bar{u}$ is an optimal solution of (P).

Proof. Since $\int_{a}^{b} f(t, \bar{x}, \dot{\bar{x}}) \mathrm{d} t=\int_{a}^{b} f(t, \bar{u}, \dot{\bar{u}}) \mathrm{d} t$

It follows from (2), (25) and (26) that

$$
\int_{a}^{b}[f(t, \bar{x}, \dot{\bar{x}})-\bar{y}(t) g(t, \bar{x}, \dot{\bar{x}})] \mathrm{d} t \leq \int_{a}^{b}[f(t, \bar{u}, \dot{\bar{u}})-\bar{y}(t) g(t, \bar{u}, \dot{\bar{u}})] \mathrm{d} t
$$

Now proceeding on the similar lines as in theorem 4.4, we get the result.

\section{Conclusions}

In this paper definition of invexity for continuous functions was extended to $\rho$-invexity of order $m$. Four types of generalizations of $\rho$-invexity of order $m$ are presented and they are:

(a) $\rho$-pseudoinvex type I functionals of order $m$,

(b) $\rho$-pseudoinvex type II functionals of order $m$,

(c) $\rho$-quasi invex type I functionals of order $m$ and,

(d) $\rho$-quasi invex type II functionals of order $m$.

An example was presented to establish that the class of $\rho$-invex functionals of order $m$ is more general than the class of $\rho$-invex functionals. Sufficient optimality conditions were established for the variational problem under $\rho$-invexity assumptions. Wolfe dual as well as Mond Weir duals was constructed. Weak, strong and converse duality results were proved using generalized $\rho$-invexity assumptions. The significance of this new notion of invexity allows us to relax the notion of invexity associated with optimality and duality results for variational problem. Further, it may be noted that the results presented in this paper are more powerful than the existing results as the class of functions deliberated here satisfies $m^{\text {th }}$ derivative test.

\section{References}

[1] Courant, R. and Hilbert, D. (1943) Methods of Mathematical Physics, 1. Wiley (Intersciences), New York.

[2] Friedrichs, K.O. (1929) Ein Verfrahen der Variations-Rechung das Minimum eines Integrals Maximum eines Anderen Ausdruckes Dazustellan, Gottingen Nachrichten.

[3] Hanson, M.A. (1964) Bounds for Functionally Convex Optimal Control Problems. Journal of Mathematical Analysis and Applications, 8, 84-89. http://dx.doi.org/10.1016/0022-247X(64)90086-1

[4] Mond, B. and Hanson, M.A. (1967) Duality for Variational Problems. Journal of Mathematical Analysis and Applications, 18, 355-364. http://dx.doi.org/10.1016/0022-247X(67)90063-7

[5] Hanson, M.A. (1981) On Sufficiency of the Kuhn-Tucker Conditions. Journal of Mathematical Analysis and Applications, 80, 545-550. http://dx.doi.org/10.1016/0022-247X(81)90123-2

[6] Mond, B., Chandra, S. and Husain, I. (1988) Duality for Variational Problems with Invexity. Journal of Mathematical Analysis and Applications, 134, 322-328. http://dx.doi.org/10.1016/0022-247X(88)90026-1

[7] Bhatia, D. and Kumar, P. (1995) Multiobjective Control Problems with Generalized Invexity. Journal of Mathematical Analysis and Applications, 189, 676-692. http://dx.doi.org/10.1006/jmaa.1995.1045

[8] Bhatia, D. and Kumar, P. (1996) Duality for Variational Problems with B-Vex Functions. Optimization, 36, $347-360$. http://dx.doi.org/10.1080/02331939608844189

[9] Husain, I. and Jabeen, Z. (2005) On Variational Problems Involving Higher-Order Derivatives. Journal of Applied 
Mathematics and Computation, 17, 433-455. http://dx.doi.org/10.1007/BF02936067

[10] Kim, D.S. and Kim, M.H. (2005) Generalized Type-1-Invexity and Duality in Multiobjective Variational Problems. Journal of Mathematical Analysis and Applications, 307, 533-554. http://dx.doi.org/10.1016/j.jmaa.2005.02.018

[11] Bhatia, G. and Sahay, R.R. (2013) Strict Global Minimizers and Higher-Order Generalized Strong Invexity in Multiobjective Optimization. Journal of Inequalities and Applications, 13, 1-14. 